\title{
Planetary nebulae abundances and stellar evolution II ${ }^{\star}$
}

\author{
S. R. Pottasch ${ }^{1}$ and J. Bernard-Salas ${ }^{2}$ \\ 1 Kapteyn Astronomical Institute, PO Box 800, 9700 AV Groningen, The Netherlands \\ e-mail: pottasch@astro.rug.nl \\ 2 Center for Radiophysics and Space Research, Cornell University, Ithaca, NY-14850-6801, USA
}

Received 7 January 2010 / Accepted 5 May 2010

\begin{abstract}
Context. In recent years mid- and far infrared spectra of planetary nebulae have been analysed and lead to more accurate abundances. It may be expected that these better abundances lead to a better understanding of the evolution of these objects.

Aims. The observed abundances in planetary nebulae are compared to those predicted by the models of Karakas (2003, Thesis, Monash Univ. Melbourne) in order to predict the progenitor masses of the various PNe used. The morphology of the PNe is included in the comparison. Since the central stars play an important role in the evolution, it is expected that this comparison will yield additional information about them.

Methods. First the nitrogen/oxygen ratio is discussed with relation to the helium/hydrogen ratio. The progenitor mass for each PNe can be found by a comparison with the models of Karakas. Then the present luminosity of the central stars is determined in two ways: first by computing the central star effective temperature and radius, and second by computing the nebular luminosity from the hydrogen and helium lines. This luminosity is also a function of the initial mass so that these two values of initial mass can be compared.

Results. Six of the seven bipolar nebulae can be identified as descendants of high mass stars $\left(4-6 M_{\odot}\right)$ while the seventh is ambiguous. Most of the elliptical PNe have central stars which descend from low initial mass stars, although there are a few caveats which are discussed. There is no observational evidence for a higher mass for central stars which have a high carbon/oxygen ratio. The evidence provided by the abundance comparison with the models of Karakas is consistent with the HR diagram to which it is compared. In the course of this discussion it is shown how "optically thin" nebulae can be separated from those which are "optically thick".
\end{abstract}

Key words. stars: abundances - planetary nebulae: general - infrared: ISM - stars: early-type - galaxies: abundances

\section{Introduction}

Planetary nebulae (hereafter PNe) are an advanced stage of stellar evolution of low and intermediate mass stars. After the asymptotic giant branch (AGB) phase is completed, these stars evolve through the PN stage before ending their lives as white dwarfs. The gaseous nebula seen now as PN is the remnant of the deep convective envelope which once surrounded the core. This core is now seen as the central star of the PN. The present abundances in the nebula reveal information about the chemical processes that took place during the AGB. These processes, which have first been discussed by Iben \& Renzini (1981) and Renzini \& Voli (1981), change the abundances according to the mass of the star involved and the initial abundances in the star. Thus by investigating the PN abundances it may be possible to assign an initial mass to the star.

Models have been made of the evolution of stars of different masses. These were initiated with the discussion of Paczynski (1971) followed by the detailed calculations of Schönberner (1983), Vassiliadis and Wood (1993) and Blöcker (1995). These models refer mostly to post AGB evolution. Models referring to evolution on the AGB have been made by several authors, e.g. Marigo et al. (2003) and Karakas (2003). The latter models predict changes in the chemical composition which have occurred during the evolution and which have been brought to the surface

^ Based on observations with ISO, an ESA project with instruments funded by ESA Member States (especially the PI countries: France, Germany, the Netherlands and the United Kingdom) and with the participation of ISAS and NASA. and subsequently expelled as the nebula. It is these models which will be used to compare with observed PN abundances because not only do they follow a star of a given mass over its entire life, but the same is done for an entire sequence of possible masses for several different initial abundances.

The purpose of the present paper is to compare these models with the abundances which we have observed. These abundances have been determined with the help of mid and far infrared observations either from ISO or Spitzer and are quite accurate because they are less affected by possible temperature variations or gradients in the nebula. These observations have already been used (Pottasch \& Bernard-Salas 2008 to better determine PN abundance gradients in the galaxy. In an ideal case it might be expected that a comparison of models with observations will lead to: 1) knowledge of the individual properties of the central stars; and 2) confirmation or suggestion for improvement of the models. In practice these goals are rather difficult to reach because of shortcomings of both the observations as well as the models. On the observational side are uncertainties in the effective temperature of the central star, their distances, as well as the accuracy of the measurements. The models presently available are uncertain because the physical conditions in the actual star-nebula system is poorly known. For example, the mass loss along the AGB (and post AGB) is physically not well understood and the initial conditions may not be realistic. Thus models used for comparison are taken from different authors who may use different mass loss rates. Therefore core masses are used where possible although initial masses are given for the Karakas models because the author identifies them as such. 
A\&A 517, A95 (2010)

Table 1. Elemental abundance of PNe with far-infrared data in addition to optical and UV data.

\begin{tabular}{|c|c|c|c|c|c|c|c|c|c|c|}
\hline $\mathrm{PNe}$ & $\mathrm{He} / \mathrm{H}$ & $\begin{array}{c}\mathrm{C} / \mathrm{H} \\
\times 10^{-4}\end{array}$ & $\begin{array}{c}\mathrm{N} / \mathrm{H} \\
\times 10^{-4}\end{array}$ & $\begin{array}{c}\mathrm{O} / \mathrm{H} \\
\times 10^{-4}\end{array}$ & $\begin{array}{l}\mathrm{Ne} / \mathrm{H} \\
\times 10^{-4}\end{array}$ & $\begin{array}{c}\mathrm{S} / \mathrm{H} \\
\times 10^{-5}\end{array}$ & $\begin{array}{c}\mathrm{Ar} / \mathrm{H} \\
\times 10^{-6}\end{array}$ & $\begin{array}{c}R^{\natural} \\
(\mathrm{kpc})\end{array}$ & Morph & Ref. \\
\hline $\mathrm{BD}+303639$ & & 7.3 & 1.1 & 4.6 & 1.9 & 0.64 & 5.2 & 7.6 & $\overline{\mathrm{R}}$ & $\mathrm{a}$ \\
\hline $\mathrm{Hb} 5$ & 0.123 & & 4.5 & 4.5 & 2.2 & 0.70 & 5.5 & 6.0 & B & b \\
\hline He 2-111 & 0.185 & 1.1 & 3.0 & 2.7 & 1.6 & 1.5 & 5.5 & 6.2 & B & $\mathrm{c}$ \\
\hline Hu 1-2 & 0.127 & 1.6 & 1.9 & 1.6 & 0.49 & 0.42 & 1.1 & 7.9 & B & d \\
\hline IC 418 & $>0.072$ & 6.2 & 0.95 & 3.5 & 0.88 & 0.44 & 1.8 & 8.8 & $\mathrm{E}$ & $\mathrm{e}$ \\
\hline IC 2165 & 0.104 & 4.8 & 0.73 & 2.5 & 0.57 & 0.45 & 1.2 & 9.8 & $\mathrm{E}$ & $\mathrm{e},{ }^{*}$ \\
\hline IC 4191 & 0.123 & & 1.49 & 7.7 & 4.7 & 1.6 & 4.45 & 7.0 & $\mathrm{E}$ & $\mathrm{p}$ \\
\hline M 1-42 & 0.161 & 10.5 & 7.5 & 8.3 & 4.4 & 2.8 & 8.6 & 3.0 & E & $\mathrm{h}$ \\
\hline Me 2-1 & 0.1 & 7.0 & 0.51 & 5.3 & 0.93 & 0.91 & 1.6 & 5.8 & $\mathrm{R}$ & $\mathrm{r}$ \\
\hline Mz 3 & $>0.080$ & $<16$ & 3.0 & 2.3 & 1.2 & 1.0 & 5.0 & 6.3 & B & $q$ \\
\hline NGC 40 & $>0.046$ & 19 & 1.3 & 5.3 & 1.4 & 0.56 & 3.4 & 7.9 & E & f \\
\hline NGC 2022 & 0.106 & 3.66 & 0.99 & 4.74 & 1.34 & 0.63 & 2.7 & 9.4 & $\mathrm{E}$ & $\mathrm{p}$ \\
\hline NGC 2440 & 0.119 & 7.2 & 4.4 & 3.8 & 1.1 & 0.47 & 3.2 & 8.9 & B & $\mathrm{g}$ \\
\hline NGC 5315 & 0.124 & 4.4 & 4.6 & 5.2 & 1.6 & 1.2 & 4.6 & 6.3 & $\mathrm{R}$ & $\mathrm{i}$ \\
\hline NGC 5882 & 0.108 & 2.2 & 1.6 & 4.8 & 1.5 & 1.3 & 2.9 & 7.2 & E & $\mathrm{e}, *$ \\
\hline NGC 6153 & 0.140 & 6.8 & 4.8 & 8.3 & 3.1 & 1.9 & 8.5 & 6.9 & E & $\mathrm{f}$ \\
\hline NGC 6302 & 0.170 & 0.6 & 2.9 & 2.3 & 2.2 & 0.78 & 6.0 & 6.4 & B & $\mathrm{j}$ \\
\hline NGC 6445 & 0.151 & 7.4: & 2.4 & 7.4 & 2.0 & 0.79 & 3.8 & 5.8 & B & $\mathrm{k}$ \\
\hline NGC 6537 & 0.149 & 1.7 & 4.5 & 1.8 & 1.7 & 1.1 & 4.1 & 6.0 & B & $\mathrm{c}$ \\
\hline NGC 6543 & 0.118 & 2.5 & 2.3 & 5.5 & 1.9 & 1.3 & 4.2 & 8.1 & $\mathrm{E}$ & $\mathrm{a}$ \\
\hline NGC 6741 & 0.110 & 6.4 & 2.8 & 6.6 & 1.8 & 1.1 & 4.9 & 6.5 & $\mathrm{E}$ & 1 \\
\hline NGC 6818 & 0.099 & 5.4 & 1.26 & 4.8 & 1.27 & 0.94 & 2.7 & 6.6 & $\mathrm{E}$ & $\mathrm{p}$ \\
\hline NGC 6886 & 0.107 & 14.3 & 4.2 & 6.5 & 2.0 & 1.0 & 2.1 & 7.7 & E & o \\
\hline NGC 7027 & 0.106 & 5.2 & 1.5 & 4.1 & 1.0 & 0.94 & 2.3 & 7.4 & E & $\mathrm{m}$ \\
\hline NGC 7662 & 0.088 & 3.6 & 0.67 & 4.2 & 0.64 & 0.66 & 2.1 & 8.2 & E & 1 \\
\hline IC 2448 & 0.094 & 2.7 & 0.55 & 2.5 & 0.64 & 0.20 & 1.2 & 8.0 & E & $\mathrm{s}$ \\
\hline NGC 2392 & 0.080 & 3.3 & 1.85 & 2.9 & 0.85 & 0.50 & 2.2 & 8.4 & $\mathrm{E}$ & $\mathrm{t}$ \\
\hline NGC 6826 & 0.10 & 4.8 & 0.58 & 3.95 & 1.5 & 0.26 & 1.4 & 8.0 & $\mathrm{E}$ & $\mathrm{u}$ \\
\hline NGC 3242 & 0.092 & 1.95 & 1.35 & 3.8 & 0.90 & 0.28 & 1.7 & 8.1 & $\mathrm{E}$ & $\mathrm{v}$ \\
\hline NGC 6369 & 0.102 & - & 0.79 & 4.0 & 0.79 & 0.60 & 1.6 & 7.2 & $\mathrm{R}$ & $\mathrm{v}$ \\
\hline NGC 6210 & 0.092 & 1.2 & 0.70 & 4.9 & 1.2 & 0.74 & 2.3 & 7.0 & $\mathrm{R}$ & w \\
\hline NGC 2792 & 0.103 & 1.1 & 0.68 & 2.1 & 0.74 & 0.43 & 1.6 & 8.2 & E & $\mathrm{x}$ \\
\hline NGC 1535 & 0.085 & 1.6 & 0.21 & 2.6 & 0.53 & 0.13 & 1.1 & 9.9 & $\mathrm{E}$ & $\mathrm{z}$ \\
\hline
\end{tabular}

Notes. ${ }^{(\mathfrak{)})}$ Galactocentric distance assuming the Sun is at $8 \mathrm{kpc}$ from the center, Morphology: $\mathrm{R}=$ round, $\mathrm{E}=$ elliptical, $\mathrm{B}=$ bipolar. ${ }^{(*)}$ Higher resolution observations.

References. a) Bernard-Salas et al. 2003, A\&A 406, 165; b) Pottasch et al. 2006; c) Pottasch et al. 2000, A\&A, 363, 767; d) Pottasch et al. 2003, A\&A, 401, 205; e) Pottasch et al. 2004, A\&A, 423, 593; f) Pottasch et al. 2003, A\&A, 409, 599; g) Bernard-Salas et al. 2002, A\&A, 387, 301; h) Pottasch et al. 2007, A\&A, 471, 865; i) Pottasch et al. 2002, A\&A, 393, 285; j) Pottasch et al. 1999, A\&A, 347, 975; k) van Hoof et al. 2000, ApJ, 532, 384; 1) Pottasch et al. 2001, A\&A, 380, 684; m) Bernard-Salas et al. 2001, A\&A 367, 949; o) Pottasch \& Surendiranath 2005, A\&A, 432, 139; p) Pottasch et al. 2005, 436, 965; q) Pottasch \& Surendiranath 2005, A\&A, 444, 861; r) Surendiranath et al. 2004, A\&A, 421, 1051; s) Guiles, S. et al. 2007, ApJ, 660, 1282; t) Pottasch et al. 2008, A\&A, 481, 393; u) Surendiranath \& Pottasch 2008, A\&A, 483, 519; v) Pottasch \& Bernard-Salas 2008, A\&A 490, 715; w) Pottasch et al. 2009, A\&A, 499, 249; x) Pottasch et al. 2009, A\&A, 502, 189; z) unpublished.

The abundances observed are listed in Table 1. No indication is given there of the spectrum of the central star. A few of these stars are Wolf-Rayet stars for which it may be that some of the evolutionary calculations may not apply. These are the central stars of BD+30 3639, NGC 40, NGC 5315 and NGC 6369. This can be kept in mind when making the comparisons.

The objects were selected to be IR bright (in the diaphragm of the instrument used). This was first done with the ISO spectrometer where almost all of the usable PN spectra were investigated. Later the Spitzer IR spectra of PNe have been investigated. Most of these spectra are as bright or nearly as bright as the ISO PNe. This may at first suggest a bias toward PNe with massive central stars because these initially evolve at the highest luminosity. But the period of high luminosity is expected to be very short so that very few, if any, high mass central star PNe are expected. We therefore may expect that many low mass central star PNe have been observed, not only because of much longer evolution time but also because of the much greater number of low mass objects present. It is expected that most of the observed $\mathrm{PNe}$ are reasonably local objects, within a few kpc of the sun.

Nevertheless a confrontation of the models with the observations, even with these limitations, can give interesting insights into the evolution of the PN system. In Sect. 2 the morphology of the nebulae will be discussed, first in relation to the nitrogen/oxygen ratio observed in the nebula, and then the helium abundance will be introduced into the discussion. In Sect. 3 the effective temperature of the central star and the various ways of determining it will be discussed. Then the luminosity of the central stars will be discussed. Because the luminosity is dependent on the distance of the nebula this will also be discussed in this section. In addition a digression will be made into the long-standing question of whether or not a nebula is "thick" to photons which ionize hydrogen. This can be done because two different methods of obtaining the nebular luminosity are 
available, one which makes no assumption concerning the nebular "thickness" and one which is dependent on this assumption. In Sect. 4 we investigate whether the expected relation between abundance and luminosity can be seen. Conclusions and discussion are given in the last section.

\section{Morphology and abundance}

There is a long history of discussion of the nebular morphology and its relation with the nebular abundance. In 1971 Greig (1971) classified the large majority of PN in two categories depending on their shape. He called these categories B (binebulous) and $\mathrm{C}$ (centric). He noted that the $\mathrm{B}$ nebulae have stronger forbidden lines of $\mathrm{N}$ II and $\mathrm{O}$ II although he did not directly relate this to abundance. Greig (1972) also noted that the B nebulae have kinematic properties indicating that they are the younger group. Several years later Peimbert (1978) classified the PN solely on the basis of the nebular abundance in four classes. His type I nebulae are nitrogen and helium rich while type II are an intermediate population having nitrogen and helium abundances close to solar. Type III, called "high velocity" does not have a substantially different composition as type II while type IV is a poorly understood group called "halo" PN which is small in number and will not concern us here.

In the five years that followed it became clear that those nebulae classified as type I also showed morphological similarities. This is summarized in an article by Peimbert \& Torres-Peimbert (1983) where many, but certainly not all, the PN listed as type I show morphology given as filamentary and bipolar. The use of abundance to indicate morphology, for example by calling a nebula type I on the basis of an abundance determination, a practice which for some years was common, led to false morphological classification. The morphological classifications listed in Col. 10 of Table 1 are based on detailed study, generally based on optical photographs, which is summarized by Manchado (2003). We also adopt the system which he gives dividing PN into three classes: bipolar (B), elliptical (E) and round (R). This system is not universally used, probably because it is not obvious that there is a fundamental difference between elliptical and round. The classification given is taken mostly from Phillips (2003) who takes this mostly, but not always, from earlier discussions in the literature. Because not all our nebulae are listed by Phillips we have also used other sources (e.g. Stanghellini et al. 1993 and Manchado et al. 1996). There is general agreement as to the classification for more than $90 \%$ of the PN but there are cases of disagreement. Stanghellini et al. have classified IC 4191, NGC 5315 and NGC 6369 irregular, but we follow all other observers (e.g. Phillips 2003) in calling them E or R. There is also speculation in the literature concerning the uncertainty introduced by projection effects but we have not attempted to include this uncertainty.

\subsection{Nitrogen/oxygen ratio}

As discussed above, we have investigated the abundances in a large number of PNe using the mid and far infrared observations from ISO or Spitzer.The resulting abundances are summarized in Table 1, which has been taken mostly from Pottasch \& Bernard-Salas (2008) but include a few new results. We regard these results as more accurate than other abundances found in the literature and have the advantage that they have been derived in the same way, which is why we have only used this sample.

A histogram of the nitrogen/oxygen (N/O) ratio for the $33 \mathrm{PN}$ listed in Table 1 is plotted as Fig. 1. Six of the seven bipolar nebulae have a very high $\log \mathrm{N} / \mathrm{O}$ ratio, between 0 and 0.4 .

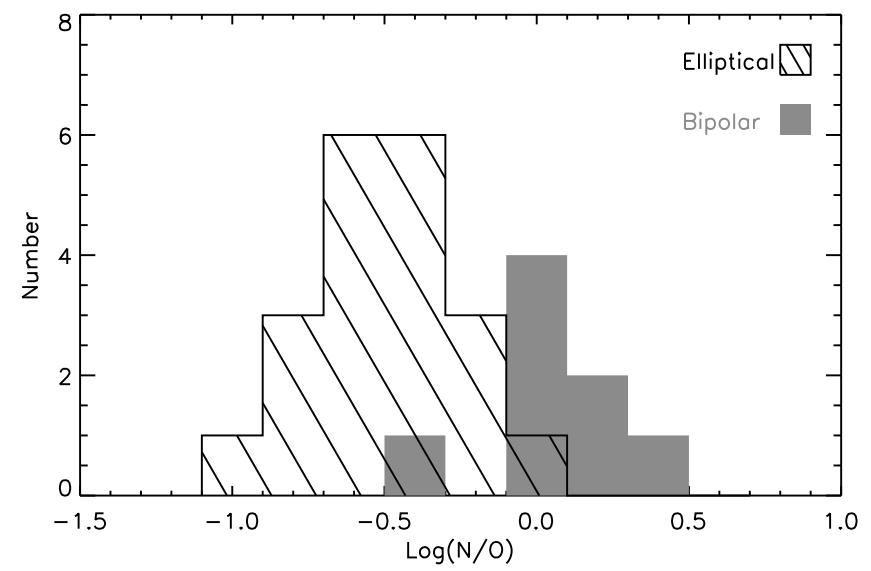

Fig. 1. Histogram of the nitrogen/oxygen ratio of the PNe listed in Table 1. Round PNe are not included in this plot because of the small number of objects.

The single exception to this is NGC 6445 which clearly has a lower value. Of the five round nebulae (not plotted), four have values of $\log \mathrm{N} / \mathrm{O}$ less than -0.66 . The fifth nebula (NGC 5315) has a much higher $\log \mathrm{N} / \mathrm{O}$ ratio $(-0.05)$, closer to that of the bipolar PN. All the elliptical nebulae have log N/O less than -0.8 , but mostly in the range -1.0 to -0.4 , with the exception of M1-42. This is a rather weak nebula and has been less well studied than the other PN. Thus we confirm the correlation between shape and $\mathrm{N} / \mathrm{O}$ ratio and show that the morphology seems to change at a $\log \mathrm{N} / \mathrm{O}$ ratio of about -0.1 .

\subsection{Helium vs. $\mathrm{N} / \mathrm{O}$}

The helium, nitrogen and oxygen abundances with respect to hydrogen are listed in Table 1 . The values of N/O are plotted in Fig. 2 as a function of $\mathrm{He} / \mathrm{H}$. The elliptical PNe are plotted as diamonds and the bipolar PNe as asterisks. It can be seen that six of the seven bipolar nebulae lie on the upper right hand side of the figure, while NGC 6445 lies somewhat by itself with a rather low N/O ratio but a rather high value of $\mathrm{He} / \mathrm{H}$. The two elliptical $\mathrm{PNe}$ which have a high $\mathrm{N} / \mathrm{O}$ ratio and thus lie close to the six bipolar nebulae are M1-42 and NGC 5315. The abundances predicted by Karakas (2003) for stars of different initial masses are labeled with numbers in the figure indicating the masses used. These masses are connected with lines for each of the three different values of heavy element abundance $Z$.

An individual comparison between the observed abundances and those predicted by Karakas is difficult because the initial values of helium and the heavy elements $(Z)$ is not known for the PNe. The value of $Z$ can be partially computed from the observed abundances but several important elements are not observed. Even for observed elements such as oxygen and carbon it is possible (although unlikely) that important amounts are tied up in dust and therefore not observable. But still important conclusions can be drawn from Fig. 2. First of all, the observed points appear to lie between the curves for $Z=0.02$ and 0.008 , i.e. there is a general agreement between the predicted and observed abundances. Four PNe seem to be in the range of $\mathrm{PNe}$ with initial mass greater than $5 M_{\odot}$. Three of them are bipolar nebulae: NGC 6302, NGC 6537 and He2-111. They appear to have passed the stage of hot-bottom burning. The fourth PN, M1-42, is difficult to compare with the theoretical curves because the high abundances indicate that it may have a high value of $Z$, and perhaps a high initial helium abundance since it lies 


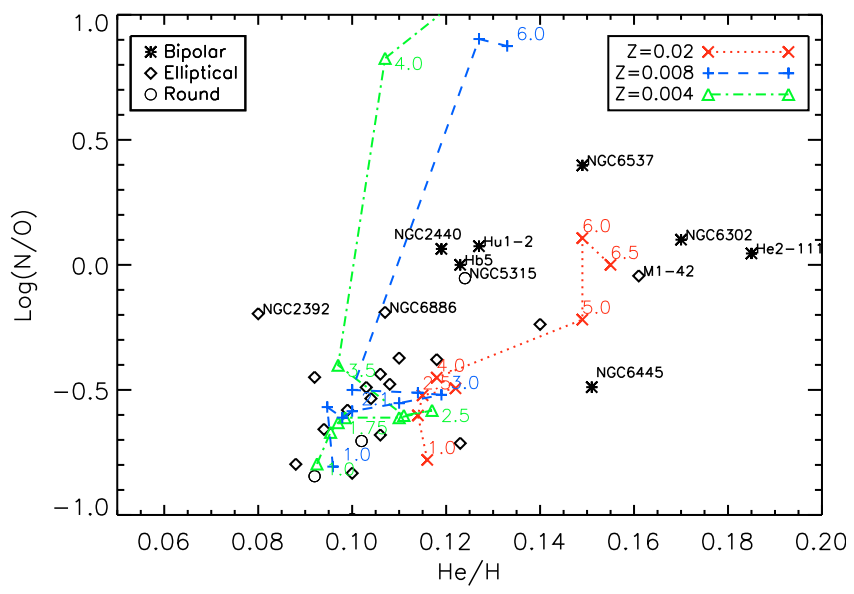

Fig. 2. The N/O abundance ratio is plotted as a function of $\mathrm{He} / \mathrm{H}$. The dashed lines connect the results of the models of Karakas for a given value of $Z$. The ciphers give the initial masses of the individual models. The individual measured PN abundances are from Table 1. Those referred to in the text also have their names given.

much closer to the galactic center than the other PNe. Three other bipolar PNe, NGC 2440, Hb5 and Hu1-2 appear to have a slightly lower initial mass, between $4 M_{\odot}$ and $5 M_{\odot}$, a value of $Z=0.008$ or somewhat higher, and have also have undergone hot-bottom burning, which destroys carbon to produce nitrogen and possibly some oxygen as well. The elliptical nebula NGC 5315 is also in this category. The three elliptical PNe NGC 6153, NGC 6886 and NGC 2392 have rather high N/O ratios indicating initial masses slightly above $4 M_{\odot}$. They have different $\mathrm{He} / \mathrm{H}$ ratios however which could indicate that they are stars of different initial helium abundances. All the other PNe are very close to the $Z=0.008$ curve for stars between initial masses of $1 M_{\odot}$ and $4 M_{\odot}$, and are all elliptical PNe. The only exception is the bipolar PN NGC 6445, whose N/O ratio indicate that it is in this initial mass range but it has a much higher $\mathrm{He} / \mathrm{H}$ ratio and therefore difficult to understand. The large majority of the nebulae can be interpreted with central star masses which agree with the helium and nitrogen abundances predicted by Karakas.

\subsection{The Carbon abundance}

The question now arises whether the observed carbon abundances fit into this picture. Carbon abundances are somewhat more uncertain than nitrogen abundances because all the observable ions are in the ultraviolet which makes them much more dependent on correct extinction and electron temperature determination. We lack four determinations of carbon in these nebulae because of the very large extinction in the ultraviolet spectrum of these nebulae which made it impossible to measure the carbon lines. In Fig. 3 the N/O ratio has been plotted against the $\mathrm{C} / \mathrm{O}$ ratio for those $\mathrm{PNe}$ with carbon abundances. The predicted values of these ratios (Karakas 2003) as a function of stellar mass for initial values of $Z=0.004,0.008$ and 0.02 are shown as points connected by dashed lines. It may be seen that a very similar picture emerges as that drawn from the $\mathrm{N} / \mathrm{O}$ vs. $\mathrm{He} / \mathrm{H}$ plot. The only three PNe which are close to the $5 M_{\odot}$ and $6 M_{\odot}$ lines are again the bipolar nebulae NGC 6302, NGC 6537 and He2-111. M1-42 now is separated from this group and is in the neighborhood of the two other bipolar PNe Hu1-2 and NGC 2440. In $\mathrm{Hb} 5$ the extinction is too high for the carbon lines to be measured. The last bipolar nebula, NGC 6445 is again at a position of lower initial mass. Again NGC 5315 and NGC 6153 are at

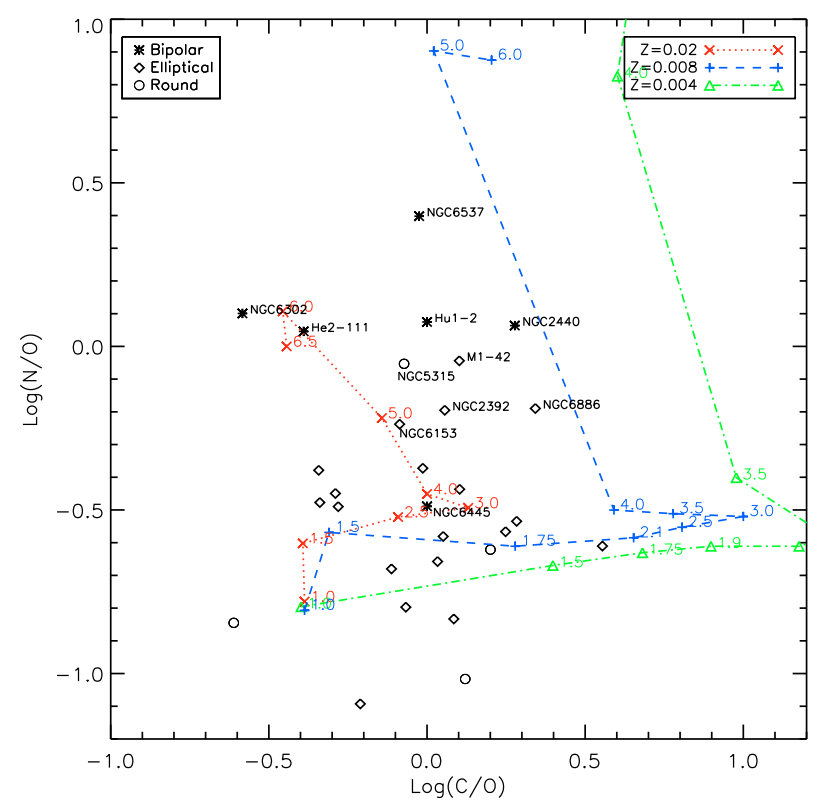

Fig. 3. N/O plotted against C/O. The lines and points are the same as in the caption to Fig. 2.

a position predicted for a mass of $4.5 M_{\odot}$, as are NGC 2392 and NGC 6886. The low helium abundance of these two PNe placed them in a more anomalous position in Fig. 2, but both the $\mathrm{N} / \mathrm{O}$ and $\mathrm{C} / \mathrm{O}$ ratios indicate that they are evolved from stars of initial mass somewhat more than $4 M_{\odot}$. NGC 6741 has about this mass as well.

The remaining PNe have lower initial mass. When the $\mathrm{C} / \mathrm{O}$ ratio greater than unity and the $\log \mathrm{N} / \mathrm{O}$ ratio is less than -0.46 , the initial mass is probably between $2 M_{\odot}$ and $3.5 M_{\odot}$. Probably the nebulae IC 418, IC 2165, NGC 40, NGC 7027, IC 2448, Me 2-1, BD+30 3639 and NGC 6826 are in this category. A $\log \mathrm{C} / \mathrm{O}$ ratio lower than -0.15 combined with a $\log$ N/O ratio less than -0.46 indicates an initial mass less than $2 M_{\odot}$. The extreme example is the central star of NGC 6210 which probably has the smallest initial mass (equal to or less than unity). Because the initial stellar abundances are not known this should only be taken as an indication.

\section{Stellar mass and luminosity}

After the thermally pulsing AGB phase terminates, low to intermediate mass stars evolve at near constant luminosity to higher temperature. The ejected envelope becomes ionized and it is seen as a planetary nebula. Models made of single star evolution through this phase (Schönberner 1983; Vassiliadis \& Wood 1993; Blöcker 1995) show that the constant stellar luminosity is a strong function of the core mass of the star. Thus determination of the luminosity provides a second method of determining the initial stellar mass which may be compared to the mass found from the abundances in the previous section. This is the purpose of the present section. First the effective temperature will be discussed, then the radius of the central star will be found using the visual magnitude of the central star. The distance of the PN plays an important role in determining the luminosity and a subsection is devoted to this subject.

The stellar luminosity can also be determined by measurements of the planetary nebula alone, even when the central star is not visible. The measurement used is either the amount of radiation which will ionize hydrogen or the amount of radiation 
Table 2. Comparison of measured stellar magnitudes.

\begin{tabular}{lrcc}
\hline \hline Nebula & $(1)$ & $(2)$ & $(3)$ \\
\hline NGC 2440 & 17.63 & 17.49 & 17.6 \\
NGC 7027 & 16.04 & 16.53 & 16.18 \\
\hline
\end{tabular}

which will doubly ionize helium. For the first case all the hydrogen ionizing radiation must be measured i.e. no ionizing radiation can escape from the nebula without causing at least one hydrogen ionization. For the second case this must be true for radiation which doubly ionizes helium. The luminosity determined in this way can be directly compared with that determined from the measurement of the central star. This is because both methods have the same dependence on the PN distance so that the distance is unimportant in the comparison. Interesting conclusions can be drawn about the "optical thickness" of the nebula as well.

\subsection{Stellar temperature}

The effective temperature of the central stars can be determined from the Zanstra method providing the spectral distribution of radiation resembles that of a blackbody and that all the photons which are able to ionize both hydrogen and ionized helium are actually absorbed, i.e. the nebula is "optically deep" for these radiation fields. This is because the Zanstra method compares the total amount of ionizing radiation (every photon which ionizes hydrogen produces a single $\mathrm{H} \beta$ photon and the $\mathrm{H} \beta$ flux is measured) with the flux in the visible part of the spectrum (as measured by the stellar visual magnitude). Consider first the stellar magnitude determination.

\subsubsection{Stellar magnitude}

The difficulty in measuring the magnitude of the central star is the result of the presence of nebular emission in the diaphragm. This must be large enough so that the star remains in the diaphragm. This is especially difficult when the star is very faint in the visible. In recent years this situation has improved: Hubble Space Telescope (HST) measurements have become available so that the image of the star is no longer broadened by the seeing. For most of the faint central stars magnitudes from the HST are now available. These are listed in the second column of Table 4. Theseare measured values and they must still be corrected for extinction. The extinction constant $\mathrm{C}$ is listed in Col. 3 and the reference in Col. 4 of the table. The accuracy can be judged by comparing the two central stars for which multiple measurements are available; these are shown in Table 2,

where Col. (1) are the HST measurements of Wolff et al. (2000), Col. (2) are HST measurements of Ciardullo et al. (1999) and Col. (3) are the ground based measurements made by Heap \& Hintzen (1990a). Very faint central stars are difficult to measure. We list the HST measurement of Matsuura et al. (2005) for NGC 6537 in Table 4 but for NGC 6302 and He2-111 the central stars are too faint or too obscured to permit measurement.

\subsubsection{Stellar radius}

Once the stellar magnitude is known, the stellar radius can be determined if the distance is known. This uncertain quantity will be discussed presently; the distances used are listed in Col. 10 of
Table 4. Further it is assumed that the stars radiate as blackbodies so that the following equation can be used:

$\left(\frac{R_{\mathrm{S}}}{d}\right)^{2}=4.808 \times 10^{-18} \times\left(\mathrm{e}^{\frac{1.439}{\lambda T}}-1\right) \times 10^{\frac{-m_{\mathrm{V}}}{2.5}}$

where $d$ is the distance, $\lambda$ is $5480 \AA, T$ is the stellar temperature and $m_{\mathrm{vo}}$ is the magnitude after correction for extinction. The resultant stellar radius is listed in Col. 11 of Table 4. The stellar temperature used is listed in Col. 8 of Table 4 and is discussed below. It does not have a large effect on the value of the radius.

\subsubsection{Zanstra temperature}

The Zanstra temperature measures the ratio of the amount of ionizing radiation to the amount of radiation in the visual spectrum. $T_{\mathrm{Z}}(\mathrm{H})$ uses the $\mathrm{H} \beta$ flux and thus measures the amount of radiation which can ionize hydrogen, while $T_{Z}(\mathrm{HeII})$ measures the amount of radiation which can completely ionize helium. In converting the ratio to a temperature it is assumed that the stellar spectrum is a blackbody, that all the ionizing radiation is absorbed by the nebula, and that the continuum visual flux is measured by the stellar magnitude.

Zanstra temperatures have been measured for many years and several papers have published extensive tables of these temperatures (e.g. Phillips 2003). The most uncertain measurement in the determination is the stellar magnitude because, as discussed above, nebular light must be avoided. The values we have found are listed in Cols. 5 and 6 of Table 4 and are not essentially different from those given in the literature. One of the most discussed aspects of the results can be seen in the table: in about $30 \%$ of the nebulae the value of $T_{\mathrm{Z}}(\mathrm{H})$ is substantially lower than $T_{\mathrm{Z}}(\mathrm{HeII})$. The reason for this has been debated in the literature; the most often cited reason is the assumption that the nebula is "optically deep" to radiation which ionizes hydrogen is wrong and that some of this radiation escapes the nebula without being registered. This has the consequence that $T_{\mathrm{Z}}(\mathrm{H})$ is too low and that $T_{\mathrm{Z}}(\mathrm{HeII})$ is the more nearly correct value. It is difficult to confirm this because not only is the total nebular mass uncertain, its distribution in the nebula is also unknown. Another explanation for this difference could also be that the stellar spectrum is not well represented by a blackbody.

\subsubsection{Energy balance temperature}

The Energy Balance method, first introduced by Stoy (1933), measures the average excess energy per ionizing photon. This can be found from the ratio of the intensity of collisionally excited nebular lines to $\mathrm{H} \beta$. It has the advantage that only the nebular spectrum has to be known; no measurement of the central star flux is necessary. It has the further advantage that it is applicable both to optically thin as well as optically thick nebulae. The method is also independent of the nebular model as long as all the collisionally excited lines are measured. In practice sometimes a correction must be made for unmeasured lines. The entire spectrum must be measured but for most PN the visible and ultraviolet lines are the most important. For very low temperature central stars the infrared nebular spectrum can be the most important.

Once the ratio of collisional line intensity to $\mathrm{H} \beta$ (called $\mathrm{R}$ ) is known a difficulty arises in interpreting this measurement in terms of a stellar effective temperature; it is necessary to know whether the star emits as a blackbody or some particular model atmosphere. Since this is not known it is assumed here that the 
star emits as a blackbody. Preite-Martinez \& Pottasch (1983) have calculated the effective temperatures found when a variety of model atmospheres of different effective temperatures are used as ionizing source. They found that for a fixed value of the ratio $\mathrm{R}$ the model atmospheres give a slightly lower value of effective temperature than the blackbody.

The exact status of the nebula also has an effect on the effective temperature found. Preite-Martinez \& Pottasch (1983) calculated three cases. In the first case the nebula is optically thin to all ionizing radiation. In the second case the nebula is optically thick to $\mathrm{He}^{+}$ionizing radiation and in the third case the nebula is optically thick to all ionizing radiation. These authors compare the effective temperatures derived for 52 central star using all of these three assumptions. They find that all three assumptions give similar results. We have redone the calculations using the case which is thick to $\mathrm{He}^{+}$ionizing radiation and thin to hydrogen ionizing radiation (case two); the effective temperatures found are listed in Col. 8 of Table 4 . As can be seen from the table, temperatures can now be found even when the central star is unobservable. The Energy balance temperature is rather similar to the HeII Zanstra temperature $T_{\mathrm{Z}}(\mathrm{HeII})$, sometimes slightly lower, sometimes slightly higher.

\subsubsection{Spectroscopic temperatures}

Stellar temperatures may also be obtained from a model atmosphere analysis of the spectrum. Because high resolution spectra are needed this has only been done for very bright stars. The results can be found in Mendez et al. (1988), Kudritzki et al. (1997) and Pauldrach et al. (2004). As Pauldrach et al. (2004) point out, the model atmosphere analysis is difficult; the results using hydrogen line profiles can differ according to which hydrogen line is used. Mendez et al. (1988) and Kudritzki et al. (1997) base their temperatures on the analysis of hydrogen and helium line profiles while Pauldrach et al. (2004) base their temperatures on the analysis of metal line profiles in the ultraviolet. The results are quite similar. The results are given in Table 3 where, considering the consistency of the different determinations, we estimate the error to be of the order of 10 to $15 \%$.

Although only six spectroscopic temperatures have been measured for our nebulae, it is interesting to compare them with what has been found from the Zanstra and Energy Balance methods. For two of the nebulae, IC 418 and NGC 6826, no $T_{\mathrm{Z}}(\mathrm{HeII})$ can be measured. In both cases there is good agreement between the spectroscopic temperature and $T_{\text {eff }}$ derived from $T_{\mathrm{Z}}(\mathrm{H})$ and $T_{\mathrm{EB}}$. In two other cases, NGC 3242 and NGC 1535, there is reasonably good agreement between the spectroscopic temperature and $T_{\text {eff }}$ derived from $T_{\mathrm{Z}}(\mathrm{HeII})$ and $T_{\mathrm{EB}}$, but definitely higher than that found from $T_{\mathrm{Z}}(\mathrm{H})$. This could also be true for IC 2448 because the spectroscopic temperature is more uncertain for this central star. It is definitely not true for the central star of NGC 2392 where both $T_{\mathrm{Z}}(\mathrm{HeII})$ and $T_{\mathrm{EB}}$ indicate a very much higher temperature. This will presently be discussed in more detail.

\subsubsection{Effective temperature}

In Col. 8 of Table 4 the estimated value of the effective temperature $T_{\text {eff }}$ is given. For those PNe where both $T_{\mathrm{Z}}(\mathrm{HeII})$ and $T_{\mathrm{EB}}$ have been measured, they are always quite similar. Of these 27 cases the average value of $T_{\text {eff }}$ is similar (within 15\%) to the hydrogen Zanstra temperature $T_{\mathrm{Z}}(\mathrm{H})$ in 15 cases. In the other 12 cases $T_{\mathrm{Z}}(\mathrm{H})$ is considerably lower than $T_{\text {eff }}$, possibly
Table 3. Spectroscopic temperatures.

\begin{tabular}{lccc}
\hline \hline Nebula & $T_{\text {eff }}{ }^{1}$ & $T_{\text {eff }}{ }^{2}$ & $T_{\text {eff }^{3}}$ \\
\hline NGC 2392 & 40000 & 45000 & 47000 \\
NGC 3242 & 75000 & 75000 & 75000 \\
IC 418 & 39000 & 37000 & 36000 \\
NGC 6826 & 44000 & 50000 & \\
NGC 1535 & & & 70000 \\
IC 2448 & & & 65000 \\
\hline
\end{tabular}

Notes. ${ }^{(1)}$ Pauldrach et al. (2004); ${ }^{(2)}$ Kudritzki et al. (1997); ${ }^{(3)}$ Mendez et al. (1988).

indicating that the nebulae are optically thin. We shall return to this subject in the next section. Four of the nebulae are not hot enough to form a substantial amount of ionized helium. In these cases substantial weight is given to $T_{\mathrm{EB}}$ which in two cases is very similar to $T_{\mathrm{Z}}(\mathrm{H})$. In the other two cases $T_{\mathrm{EB}}$ is higher than $T_{\mathrm{Z}}(\mathrm{H})$ and an average of the two temperatures is used. For $\mathrm{Mz} 3$ the temperature is determined from the best fitting nebular model (see Pottasch \& Surendiranath 2005c). The value of $T_{\text {eff }}$ for NGC 6302 is discussed below.

\subsection{PN distances}

Distances to PNe are uncertain because the usual method for astronomical distance determination, parallax, is applicable to only a very limited number of nebulae. In this situation two options are available. One can use less accurate methods for determining individual distances or one can assume that all nebulae have a particular property in common and use this property to obtain a statistical distance. There is an extensive literature for determining distances with the assumption that all nebulae have the same ionized mass. Especially the work of Cahn et al. (1992) or more recently the work of Stanghellini et al. (2008), which calibrates the mass using Magellanic Cloud PNe measurements, have been used. Both these determinations assume that the $\mathrm{PNe}$ are optically thin to hydrogen ionizing radiation but attempt to correct this assumption for the smaller high density nebulae.

The statistical distances will not be used here because PNe evolve from stars of a wide range of stellar masses and we do not wish to exclude the possibility that PNe of different stellar mass produce nebulae with different properties. Averaging any of these properties may lead to systematic errors in the individual distances found from these average properties. This in turn could introduce systematic errors in evolution calculations made using them. In addition Ciardullo et al. (1999) measured accurate distances of a small sample of binary PNe and concluded that for these nebulae the statistical distances are overestimates. Instead we determine distances using three methods which, while giving somewhat more uncertain individual distances, are much less likely to have systematic errors.

These methods are well known and are long in the literature. The first method is the expansion distances where the expansion is measured at two epochs, usually separated by 3 to 5 years. The measurements are made in both the optical (HST measurements are desirable) and at radio frequencies (VLA measurements are usually used). Expansion velocities must also be known but they cannot be measured in the plane of the sky in which the expansion is observed. The measured radial velocities are therefore used in the hope that these are very similar. In addition there may be gradients in the velocity which must be taken into account. Because the nebular density decreases between the two 
Table 4. Central star magnitude, temperature and luminosity.

\begin{tabular}{|c|c|c|c|c|c|c|c|c|c|c|c|c|c|}
\hline $\mathrm{PNe}$ & $\begin{array}{c}m_{V} \\
\text { meas. }\end{array}$ & $\begin{array}{l}\mathrm{C} \\
(3)\end{array}$ & $\begin{array}{l}\text { Ref. } \\
(4)\end{array}$ & $\begin{array}{c}T_{\mathrm{Z}}(\mathrm{H}) \\
(5)\end{array}$ & $\begin{array}{c}T_{\mathrm{Z}}(\mathrm{HeII}) \\
(6)\end{array}$ & $\begin{array}{l}T_{\mathrm{EB}} \\
\text { (7) }\end{array}$ & $\begin{array}{l}T_{\text {eff }} \\
(8)\end{array}$ & $\begin{array}{r}\mathrm{H} \beta \\
\times 10^{11}\end{array}$ & $\begin{array}{l}\text { dist } \\
\mathrm{kpc}\end{array}$ & $\begin{array}{c}R_{\mathrm{S}} / R_{\odot} \\
(11)\end{array}$ & (12) & $\begin{array}{c}L_{\mathrm{S}} / L_{\odot} \\
(13)\end{array}$ & (14) \\
\hline$\overline{B D}+30$ & 10.28 & 0.50 & 9 & 31500 & & 30000 & 30000 & 29.5 & 1.0 & 1.66 & 1870 & 2450 & \\
\hline $\mathrm{Hb} 5$ & 17.1 & 1.60 & 8 & 105000 & 130000 & 145000 & 140000 & 11.9 & 2.3 & 0.128 & 5600 & 2700 & 4550 \\
\hline He $2-111$ & & & & & & & 200 000: & 1.34 & 2.5 & & & & 650 \\
\hline Hu 1-2 & 17.76 & 0.60 & 6 & 100000 & 145000 & 130000 & 140000 & 2.22 & 1.5 & 0.0335 & 390 & 210 & 540 \\
\hline IC 418 & 10.23 & 0.33 & 1 & 34000 & & 36000 & 35000 & 58.3 & 1.0 & 1.3 & 1850 & 3700 & \\
\hline IC 2165 & 17.47 & 0.59 & 2 & 13200 & 135000 & 120000 & 134000 & 4.98 & 1.5 & 0.0774 & 1750 & 1810 & 2680 \\
\hline IC 4191 & 16.4 & 0.48 & 11 & 79000 & 90000 & 97000 & 90000 & 5.25 & 2.1 & 0.099 & 580 & 750 & 1300 \\
\hline M 1-42 & 17.4 & 0.63 & $\mathrm{~h}$ & 63000 & 81000 & 100000 & 85000 & 1.0 & 5.0 & 0.179 & 1500 & 810 & 1690 \\
\hline Me 2-1 & 18.40 & 0.28 & 2 & 130000 & 145000 & 142000 & 145000 & 0.935 & 2.3 & 0.0273 & 300 & 210 & 480 \\
\hline Mz 3 & & 1.65. & & & & & 39500 & 29.6 & 1.3 & & & 2300 & \\
\hline NGC 40 & 11.55 & 0.605 & 1 & 35000 & & 40000 & 38000 & 17.3 & 0.8 & 0.683 & 875 & 570 & \\
\hline NGC 2022 & 15.75 & 0.38 & 111 & 59000 & 108000 & 105000 & 108000 & 1.79 & 1.8 & 0.099 & 1200 & 220 & 1500 \\
\hline NGC 2440 & 17.63 & 0.50 & 2 & 208000 & 205000 & 18000 & 200000 & 9.1 & 2.0 & 0.037 & 2000 & 1900 & 2100 \\
\hline NGC 5315 & 14.3 & 0.54 & 3 & 65000 & 76000 & 66000 & 70000 & 13.3 & 2.0 & 0.306 & 2020 & 1720 & \\
\hline NGC 5882 & 13.42 & 0.33 & 1 & 50000 & 64000 & 70000 & 67000 & 12.7 & 1.2 & 0.231 & 979 & 590 & 1070 \\
\hline NGC 6153 & 15.55 & 1.14 & 1 & 77000 & 87000 & 80000 & 82000 & 21.5 & 1.2 & 0.169 & 1200 & 1000 & 2000 \\
\hline NGC 6302 & & & & & & 300 000: & 300.000: & 56. & 1.6 & & & 8000 & 7400 \\
\hline NGC 6445 & 18.72 & 1.06: & 3 & 185000 & 175000 & 175000 & 180000 & 8.7 & 1.8 & 0.0348 & 1200 & 1380 & 1170 \\
\hline NGC 6537 & 21.6 & 1.79 & 5 & 410000 & 350000 & & $350000:$ & 12.8 & 2.0 & 0.0147 & 2950 & 4200 & 3700 \\
\hline NGC 6543 & 11.29 & 0.10 & 1 & 47000 & & 58000 & 56000 & 30.8 & 1.0 & 0.457 & 1840 & 1050 & \\
\hline NGC 6741 & 20.09 & 1.1 & 4 & 205000 & 219000 & 230000 & 220000 & 4.1 & 1.9 & 0.184 & 710 & 850 & 400 \\
\hline NGC 6818 & 17.02 & 0.35 & 3 & 140000 & 155000 & 138000 & 145000 & 7.43 & 2.0 & 0.048 & 940 & 1270 & 2150 \\
\hline NGC 6886 & 18.76 & 0.70 & 6 & 145000 & 145000 & 156000 & 152000 & 2.34 & 2.6 & 0.038 & 680 & 670 & 690 \\
\hline NGC 7027 & 16.07 & 1.3 & 210 & 165000 & 160000 & 234000 & 180000 & 134. & 0.9 & 0.0716 & 4820 & 5300 & 4100 \\
\hline NGC 7662 & 14.00 & 0.18 & 1 & 80000 & 108000 & 95000 & 103000 & 15.3 & 1.2 & 0.118 & 1400 & 770 & 2500 \\
\hline IC 2448 & 14.26 & 0.27 & 1 & 48000 & 83000 & 91400 & 85000 & 2.35 & 1.4 & 0.147 & 1160 & 151 & 850 \\
\hline NGC 2392 & 10.63 & 0.22 & 1 & 37000 & 78000 & 80000 & 80000 & 6.83 & 1.5 & 0.845 & 26000 & 490 & 4450 \\
\hline NGC 6826 & 10.68 & 0.07 & 1 & 34000 & & 45000 & 42000 & 12.8 & 1.4 & 0.98 & 2680 & 1120 & \\
\hline NGC 3242 & 12.32 & 0.12 & 12 & 57000 & 90000 & 70000 & 80000 & 21.4 & 0.55 & 0.14 & 740 & 260 & 1600 \\
\hline NGC 6369 & 15.91 & 2.12 & 312 & 69000 & 71000 & 70000 & 70000 & 61.6 & 1.2 & 0.40 & 3250 & 2850 & 2900 \\
\hline NGC 6210 & 12.66 & 0.14 & 7 & 51000 & 61000 & 69000 & 65000 & 11.0 & 1.57 & 0.36 & 2070 & 890 & 1060 \\
\hline NGC 2792 & 16.89 & 0.80 & 111 & 82000 & 135000 & 126000 & 130000 & 3.05 & 1.9 & 0.079 & 1600 & 430 & 1570 \\
\hline NGC 1535 & 12.11 & 0.08 & 1 & 40000 & 76000 & 87000 & 80000 & 4.8 & 2.1 & 0.519 & 9900 & 680 & 2500 \\
\hline
\end{tabular}

Notes. $\mathrm{H} \beta$ in units erg $\mathrm{cm}^{-2} \mathrm{~s}^{-1}$, Col. 7 is the energy balance temperature, Col. 8 is the effective temperature, Col. 12 1s the luminosity found from the magnitude and effective temperature of the central star, Col. 13 is the luminosity found from the nebular hydrogen line, Col. 14 is the luminosity found fron the nebulae ionized helium line.

References. 1) Ciardullo et al. (1999); 2) Wolff et al. (2000); 3) Gathier \& Pottasch (1988); 4) Sabbadin et al. (2005); 5) Matsuura et al. (2005); 6) Heap et al. (1990b); 7) Pottasch et al. (2009); 8) Tylenda et al. (2003); 9) Crowther et al. (2006); 10) Zijlstra et al. (2008); 11) Preite-Martinez et al. (1991); 12) Pottasch \& Bernard-Salas (2008).

measured epochs, the ionization front appears to move more quickly than the matter and this must also be taken into account. A discussion with many results is given by Terzian (1997) and the effect of the ionization front is discussed by Mellema (2004). In addition, Schönberner et al.(2005) have made and applied kinematic models of several PNe to obtain expansion distances. There is general agreement for the four PNe common to the results of both Mellema(2004) and Schönberner et al.(2005), which indicate some of the expansion distances given by Terzian (1997) should be increased. The increases depend on the individual models and are thus uncertain. Both authors estimate that the results Terzian (1997) should on average increase by about $30 \%$. These possible increases are taken into account in our distance estimates, although other methods, especially extinction distances, may place important limits on the increase. For the PNe we are discussing 9 expansion distances are known (NGC 3242, NGC 6210, NGC 6302, NGC 6543, NGC 7027, NGC 7662, IC 418, IC 2448 and BD+30 3639).

The second method is the extinction distance. In this method the extinction of field star of known distance located within a small distance from the nebula in the plane of the sky (usually less than 0.5 degrees) is measured and a plot is made of reddening as a function of distance in that direction. The reddening of the PNe is placed on this diagram and the distance read off. This method assumes that the extinction is rather uniform over the 0.5 degrees (or smaller) used, and that the PNe is located in the galactic plane where the extinction increases with distance. This is a rather time consuming process. It has been done for NGC 2440, NGC 2792, NGC 5315, NGC 5882, NGC 6543, NGC 6741, NGC 7027 and IC 2448 among the PNe we are interested in (see Gathier et al. 1986a; Martin 1994).

There is a variant of this method which instead of measuring the extinction of the PN and nearby sources, measures the $21 \mathrm{~cm}$ neutral hydrogen radio line absorption of the PN and nearby sources. This method has the advantage that the velocity of the absorption line is measured so that it may be ascertained whether the nebula is on the near or far side of a particular spiral arm. This method has been used for NGC 6369, NGC 6537, NGC 6886 and NGC 7027 (of the PNe which interest us). This method is again limited to PNe near the galactic plane (see Gathier et al. 1986b).

In addition use can be made of the average extinction (in magnitudes per kpc) in various directions. Such maps have been made by several authors but because a relatively limited number 
of stars are used, these maps are averages over relatively large areas and are more uncertain than when a small area is studied in depth. Results of this method are given for a large number of PNe by Pottasch (1984) and Sabbadin (1986). The results of these two authors generally agree when the same nebulae are compared. We will use them when no other individual distance is available.

Another method of distance determination is from the spectroscopic measurements of the central star. These measurements have already been discussed in Sect. 3.1.5 for determining the central star temperatures for which, with the exception of NGC 2392, reasonable agreement was obtained with the temperatures found from the nebular spectrum. The same discussions (Pauldrach et al. 2004; Kudritzki et al. 1997; and Mendez et al. 1988) derive the distance and mass of various bright central stars. These distances are not included in the present discussion. The reason for this is the following. The large masses and distances found by Pauldrach et al. are improbable. This has been convincingly demonstrated by Napiwotzki (2006), who found that the kinematic properties of these nebulae are inconsistent with the masses and distances given by these authors. This reasoning can be extended to the distances found by Kudritzki et al. which are very similar. Some, but not all, of the distances found by Mendez et al. (1992) are similar to those given by Pauldrach et al. and Kudritzki et al. There are six PNe in common between Mendez et al. and those used by us. For one PN, NGC 1535, the same distance is given. For two other nebulae, IC 2448 and NGC 3242 there is a strong discrepancy between the expansion distance as given by Mellema (2004) or Schönberner et al. (2005) in the sense that Mendez et al. give values of 3.5 and $1.8 \mathrm{kpc}$ while the expansion distance is considerably lower, 2.1 and $0.55 \mathrm{kpc}$ respectively. For the remaining 3 PNe, NGC 6826, NGC 2392 and IC 418 our individual distances are about $45 \%$ smaller than those given by Mendez et al. (1992). For the single PN for which an optical parallax is available, NGC 7293, Harris et al. (2007) measure a distance of $219 \mathrm{pc}$ while Mendez et al. (1992) give the higher value of $300 \mathrm{pc}$. We regard the values of Mendez et al. as rather high and do not give tham much weight.

Distances may also be determined from the nebular spectrum. When making a model of the nebula to explain, not only the relative intensity of the lines relative to hydrogen, but the absolute hydrogen line intensities as well, the distance of the nebula is one of the unknown quantities (along with the stellar radius and temperature and the nebular density and temperature). Our experience with such models indicate that the distances determined in this way have an accuracy of about $20 \%$. Model distances are available for NGC 2792, NGC 6826, NGC 6886, NGC 6741, NGC 6445, Hu1-2, Hb5, Me2-1 and Mz3. In addition the distance to NGC 1535 has been measured by the fact that it is a double star and the companion has a spectroscopic parallax.

In this way individual distances are known for 32 of the $33 \mathrm{PNe}$ being studied. The only exception is He2-111 for which a statistical distance has been used. We feel that the distances listed in Table 4 are an improvement over the older ones shown in our previous paper.

\subsubsection{Comparison with statistical distances}

In the above we have discussed the reason for not using the statistical distances. Here we compare the individual distances with the statistical distances found in the literature. There are two reasons for doing this. First, to show that on average the statistical distances are not very different from the individual distances.
This is to counter a possible remark when the nebular luminosities are discussed, that increasing PNe distances by a large amount is a reasonable alternative. Secondly we wish to demonstrate that bipolar PNe are affected in a different way than elliptical PNe when statistical distances are determined, thus demonstrating the bias discussed above.

There are many statistical distance scales in the literature and for clarity only two of them will be discussed here. These are the often used scale of Cahn et al. (1992, hereafter CKS) who calibrate distances using "well known" PNe distances, and the more recent distances of Stanghellini et al. (2008, hereafter SVV) who make use of the PNe in the Large Magellanic Cloud as calibrators. Both scales assume that all PNe are optically thin to hydrogen ionizing radiation and have the same ionized mass, but in both scales a correction to this assumption is made for nebulae considered optically thick. The differences between the distances given by these two sources is usually not large.

Of the $32 \mathrm{PNe}$ (excluding He2-111 for which no individual distance is available), the individual distances to 20 nebulae are found to be the same as the statistical given by CKS, 8 PNe have larger individual distances and $4 \mathrm{PNe}$ have smaller individual distances. Compared to the distances given by SSV, 14 PNe have the same distance, 2 PNe are larger than given by SSV and 11 PNe have smaller distances. Having the same distance is defined as being within $33 \%$ of the larger of the two distances. Note that in the comparison with SVV there are slightly less PNe in common.

But if only the bipolar nebulae are considered a somewhat different picture emerges. Of the 7 bipolar nebulae (again excluding He2-111) the distances to 5 of them are larger than given by CKS, 2 are at the same distance and there are no PNe at smaller distances. For SVV these numbers are 2, 3 and 0 respectively. Thus while the individual distances are rather similar to the statistical distances for the whole sample, they are on the whole larger than the statistical distances for the bipolar nebulae. This illustrates the danger of using statistical distances which averages out the differences in different classes of nebulae. For the two PNe which on the basis of the nebular abundance evolve from the most massive stars, NGC 6537 and NGC 6302, we find distances twice as high as given by CKS or SSV.

\subsection{Luminosity}

\subsubsection{Luminosity from central star.}

Once the effective temperature $T_{\text {eff }}$, the stellar radius, and the distance to the PNe are known the luminosity can be calculated. Using the relation:

$$
L_{\mathrm{S}} / L_{\odot}=\left(R_{\mathrm{S}} / R_{\odot}\right)^{2} \times\left(T_{\mathrm{eff}} / T_{\odot}\right)^{4}
$$

the stellar luminosity given in Col. 12 of Table 4 is found. It is difficult to estimate the error of this luminosity. The distance is not well known so that the error could be as much as $30 \%$; for individual objects it might be even higher. This introduces an error of a factor of two in the luminosity and is probably the largest error. The stellar magnitude and extinction value are probably reasonably well known and are not likely to introduce an error of more than $20 \%$ unless the wrong star has been measured. We shall return to this presently. The effective temperature, which is related to the assumption of blackbody radiation, may also be in error. An error of $10 \%$ in temperature leads to an uncertainty of $40 \%$ in luminosity. 


\subsubsection{Luminosity from nebular emission}

The luminosity can also be determined from the emission lines of either hydrogen or ionized helium, at least when the nebula absorbs all the ionizing radiation. Consider hydrogen: every photon with energy greater than $13.6 \mathrm{eV}$ emitted by the central star ionizes one hydrogen atom and then produces a single $\mathrm{H} \beta$ photon. Thus the $\mathrm{H} \beta$ luminosity can be converted to the total luminosity of the star above $13.6 \mathrm{eV}$. When the temperature is known this can be converted to the total stellar luminosity. This may be written as:

$L_{\mathrm{S}} / L_{\mathrm{H} \beta}=C_{1} \times T / G_{1}(T)$

where

$C_{1}=\frac{\pi^{4} k \alpha_{\mathrm{B}(\mathrm{H})}}{15 h \nu_{\mathrm{H} \beta} \alpha_{\mathrm{H} \beta}}$

$L_{\mathrm{H} \beta}=4 \pi d^{2} F_{\mathrm{H} \beta}$

and $\mathrm{G}_{1}(\mathrm{~T})$ is an integral shown as Eq. (VII-8) whose values are tabulated in Table VII-4 by Pottasch (1984). $F_{\mathrm{H} \beta}$ is listed in Col. 9 of Table 4. The luminosity computed in this way has already been used by Pottasch \& Acker (1989). It is similar to the Zanstra method in the assumptions as well as in the equations. Like the Zanstra method the luminosity can also be computed from the $\lambda 4686 \AA$ line of ionized helium. The equations then become:

$L_{\mathrm{S}} / L_{4686}=C_{2} T / G_{4}(T)$

where

$C_{2}=\frac{\pi^{4} k \alpha_{\mathrm{B}\left(\mathrm{He}^{+}\right)}}{15 h v_{4686} \alpha_{4686}}$

$L_{\mathrm{He} 4686}=4 \pi d^{2} F_{4686}$

where the references are the same as above.

The function $T / G_{x}(T)$ has a minimum value for both hydrogen and ionized helium. For hydrogen the function does not change by more than $25 \%$ between temperatures of $45000 \mathrm{~K}$ and $150000 \mathrm{~K}$. Within this range small errors in the temperature will have only a reasonably small effect on the luminosity. Outside of this range, and especially at the lower temperatures, an error in the temperature will have a much larger effect. This should be taken into account when comparing the luminosities determined in the different ways. For the luminosities found from the ionized helium line, the range of temperature where the effect is small is between $140000 \mathrm{~K}$ and $800000 \mathrm{~K}$. Again a small error in temperature will have a much large effect at lower temperatures. This is illustrated in the PN NGC 6302. The central star, which is not visible, has an uncertain energy balance temperature of 300000 K (Preite-Martinez \& Pottasch 1983). The luminosity computed from the $\lambda 4686$ line is $7400 L_{\odot}$. This value is only slightly dependent on the temperature between $200000 \mathrm{~K}$ and $500000 \mathrm{~K}$. The value found from the $\mathrm{H} \beta$ line is much more sensitive to the temperature and only at a value close to $300000 \mathrm{~K}$ can the same luminosity be computed. This fixes the temperature.

The luminosities found from the $\mathrm{H} \beta$ line are listed in Col. 13 of Table 4 , and the luminosities found the $\lambda 4686$ line are given in Col. 14 of the same table.

\subsubsection{Comparison of stellar and nebular luminosities}

Both the stellar and nebular luminosities have the same dependence on the distance so that a comparison of the two does not involve the distance.

On making this comparison the general impression is of reasonably good agreement between the stellar and nebular luminosities. On closer inspection we can distinguish several cases. Case 1 are those PNe where the stellar luminosity (Col. 12 in Table 4) and the $\lambda 4686$ luminosity (Col. 14) agree and are both higher than the $\mathrm{H} \beta$ luminosity. We regard these $\mathrm{PNe}$ as optically thin to hydrogen ionizingradiation and optically thick to radiation which doubly ionizes helium. For these cases we use the average of Cols. 12 and 14 as the luminosity of the exciting star. There are eight cases: Hb5, Hu1-2, M1-42, Me2-1, NGC 2022, NGC 5882, IC 2448 and NGC 2792. In case 2 are those nebulae where all three luminosities agree. These are regarded as optically deep to both hydrogen and doubly ionized helium. For these eight cases the average value of all three luminosities is used. These are NGC 2440, NGC 5315, NGC 6445, NGC 6537, NGC 6886, NGC 6369, NGC 6741 and NGC 7027. Then there are those nebulae (case 3 ) where the stellar luminosity agrees with that determined from the $\mathrm{H} \beta$ luminosity, while that determined by the doubly ionized helium luminosity is about a factor of two higher. This is probably due to the use of a slightly too low effective temperature, since in all cases the temperature dependence of the luminosity is quite large. The PNe involved are IC 2165, IC 4191, NGC 6153 and NGC 6818. Here the stellar luminosity is used (Col. 12). These PNe are considered optically thick to all ionizing radiation fields. It is likely that NGC 7662 and NGC 3242 should be included in this group because here again the doubly ionize helium luminosity is about twice the stellar luminosity, but because these nebulae are optically thin to hydrogen ionizing radiation the hydrogen luminosity is lower. Case 4 are the five $\mathrm{PNe}$ which have low temperature central stars and thus do not have a HeII luminosity. Since these nebulae have low temperature central stars the nebular luminosity is a very strong function of the temperature. For these five PNe (BD+30 3639, IC 418, IC 40, NGC 6543 and NGC 6826) we use an average luminosity giving double weight to luminosity determined from the star.

We have now discussed 30 of the 33 PNe. For NGC 6210 the stellar luminosity is twice the nebular luminosity. We will use an average value of the three luminosities listed. The problem concerning NGC 2392 is greater. The stellar luminosity for this object is an order of magnitude greater than the nebular luminosity. It is also much greater than for all the other nebulae. The problem probably lies with the temperature we have assigned to this star. As discussed in Sect. 3.1.5 and Table 4 the spectrum of this star is not more than $50000 \mathrm{~K}$, much less the value of $80000 \mathrm{~K}$ which has been used. But a blackbody of $50000 \mathrm{~K}$ does not have enough ultraviolet radiation to produce the observed nebular spectrum. Thus there must be an additional source of ultraviolet radiation. This could be another hotter star. This has been suggested several times in the literature because of the incompatibility of the nebular and stellar spectrum. Ciardullo et al. (1999) have examined HST photographs of this nebula and have found a faint star at 2.65 " from the bright "central star". This star is only seen through the red $(I)$ filter; it is invisible in the visible $(V)$. It is therefore likely to be a red star unless it has a very unusual spectrum. In any case, the stellar luminosity listed for this star in Table 4 is certainly not correct. If one wishes to assign a luminosity to the exciting star, we suggest using the nebular 
luminosity found from the $\lambda 4686$ line. The PNe is clearly optically thin to radiation capable of ionizing hydrogen.

The luminosities for NGC 1535 show a great similarity to those of NGC 2392. Again the stellar luminosity is considerably higher than the nebular luminosities and the nebula is optically thin to radiation capable of ionizing hydrogen. But in this case the effective temperature which we have assigned to the central star is not very different from the spectroscopic temperature shown in Table 3 (although only a single determination is available). It is possible that in this case the nebula is optically thin to radiation capable of doubly ionizing helium. We find it difficult to assign a luminosity to this central star.

There are $25 \mathrm{PNe}$ which have both hydrogen and ionized helium Zanstra temperatures. We define the ratio $T_{Z}(\mathrm{HeII}) / T_{Z}(\mathrm{H})$ as the Zanstra ratio. Ignoring NGC 6210, there are 12 optically thick PNe and 12 optically thin PNe as found above by a consideration of their luminosities. The Zanstra ratio for the 12 optically thick PNe varies between 0.95 and 1.17 with a median value of 1.03 . For the 12 optically thin PNe the Zanstra ratio varies between 1.12 and 2.1 with a median value of 1.65. We conclude the assumption that the star radiates as a blackbody is reasonable and consistent. Furthermore those PNe with a Zanstra ratio greater than 1.2 are optically thin, while those lower than 1.1 are optically thick. Between values 1.1 and 1.2 further information is necessary.

\subsection{Core mass and the HR diagram}

In the earlier discussions of the work of Karakas (2003) her various models have been identified by their initial mass, just as Karakas has done. A more direct mass to use in discussing the HR diagram is the core mass, as it avoids uncertainties in the assumed mass loss. The $Z=0.008(Z=0.02)$ models of Karakas labeled with the initial masses of $1,1.5,2.5,4.0$ and $6.0 M_{\odot}$ have core mass values of $0.60(0.57), 0.63(0.60), 0.66(0.66)$, $0.84(0.79)$ and $0.95(0.93) M_{\odot}$. The values of $Z$ for the PN considered can be calculated from the abundances in Table 1 . The abundances are in general rather similar to the solar abundances as given by Asplund et al. (2005). These authors calculate the solar valule of $Z=0.0134$. Thus most of the $Z$ values calculated from Table 1 lie between 0.008 and 0.02 in approximate agreement with their position in Figs. 2 and 3.

In this section the luminosities determined above are used to compare with the luminosities other models predict in order to see if they agree with the core masses given by Karakas. It would have been more consistent to compare with tracks of the original models of Karakas but she has not given post AGB tracks for these models.

The effective temperature and the luminosity of the central stars shown in Table 4 are plotted as points in Fig. 4. The individual $\mathrm{PN}$ is identified on the left side of the diagram. Several different models are plotted as solid lines on the figure. The core mass and initial heavy element abundance $\mathrm{Z}$ used in the model is shown in the diagram. Not shown on the diagram are the phase of the helium shell flash cycle at which the ejection is assumed to occur and the mass loss rate used in making that model. The mass loss rate in particular is a very poorly known quantity and can result in large uncertainties in the models. In Fig. 4 the results of Vassiliadis \& Wood (1993) are used for the core mass $M=0.57 M_{\odot}, M=0.67 M_{\odot}$ and $0.91 M_{\odot}$ models (the first has $Z=0.016$, the two other models have $Z=0.008$ ), and the results of Schönberner (1983) for the $M=0.55 M_{\odot}$ model.
As can be seen in the diagram, two of the three PNe (NGC 6537 and NGC 6302) for which it was concluded on the basis of their abundance that they originate from high mass stars, indeed are in the high mass region of the HR diagram. The third $\mathrm{PN}$ (He2-111) might also be in this region but the temperature determination is too uncertain to be sure of this. The three other bipolar PNe, NGC 2440, Hb5 and Hu1-2 whose abundance indicated a somewhat lower core mass, are in a rather higher mass portion of the HR diagram. The precise value of the initial mass agrees less well, since on the basis of the abundances we predicted in Sect. 2 that these PNe originated from an initial mass of $4 M_{\odot}$ to $5 M_{\odot}$ which following Karakas (2003) corresponds to a core mass of slightly higher than $0.8 M_{\odot}$ while in the HR diagram they are in the position of the $0.67 M_{\odot}$ track. In addition several other PNe lie in the same position. NGC 6886 has a high N/O ratio and on this basis was suspected to originate from a higher mass star. The bipolar PN NGC 6445, which because of its high $\mathrm{He} / \mathrm{H}$ and low N/O ratios was considered enigmatic, is also in this region. But so are NGC 7027 and NGC 6741 whose abundances would place them in the group of low initial mass objects. Also enigmatic are NGC 5315 and M1-42 which on the basis of both high $\mathrm{He} / \mathrm{H}$ and $\mathrm{N} / \mathrm{O}$ ratios might be expected to have somewhat higher core masses, are in the region of low mass objects on the HR diagram.

On the other hand, the majority of PNe which on the basis of their abundances are thought to have evolved from low mass stars, do indeed fall in the low mass region of the HR diagram. We have checked to see whether those PNe which are carbon rich $(\mathrm{C} / \mathrm{O} \geq 1)$ have a special position on the HR diagram, but we find them to be at positions indistinguishable from other low initial mass objects.

To summarize, there is a qualitative agreement between the two ways of approaching stellar evolution: by either looking at the nebular abundances or the position of the central star on the $\mathrm{HR}$ diagram. The three bipolar PNe whose high N/O and $\mathrm{He} / \mathrm{H}$ leads to the prediction (Karakas 2003) that they have core masses of $0.85 M_{\odot}$ to $0.95 M_{\odot}$ are in a position on the HR diagram that is consistent with the theoretical $0.9 M_{\odot}$ tracks of Vassiliadis \& Wood (1993) or the $0.91 M_{\odot}$ tracks of Blöcker (1995) (not shown in Fig. 4). The other three bipolar PNe with somewhat lower abundances and where the predictions of Karakas lead us to expect a slightly lower core mass, probably between $0.8 M_{\odot}$ and $0.88 M_{\odot}$, show less good agreement with the model tracks. In Fig. 4 these PNe agree with the $0.67 M_{\odot}$ tracks of Vassiliadis \& Wood (1993) but they also agree with one of the $0.6 M_{\odot}$ tracks of Blöcker (1995).

Regarding the elliptical PNe: many of the central stars fall near the tracks of stars of core mass between $0.55 M_{\odot}$ and $0.57 M_{\odot}$ on the HR diagram. Stars of these masses are not expected to show abundance changes which are at present observable, in agreement with the observations. Karakas (2003) predicts a core mass of $0.6 M_{\odot}$ for these PNe, more in line with the value of $0.58 M_{\odot}$ expected from the average measured white dwarf masses. The reason that the observed luminosity does not agree with that predicted from the models is not clear. If the observed luminosity were too low, the most likely cause would be a possible underestimate of the observed PNe distance. To obtain agreement with the model luminosity the average distance would have to be increased by $70 \%$. We regard this as unlikely for an average value, although it may occasionally occur for individual PNe.The other possibility is that the relationship between core mass and luminosity found for the low core mass models is not entirely correct. 
S. R. Pottasch and J. Bernard-Salas : Planetary nebulae abundances and stellar evolution II

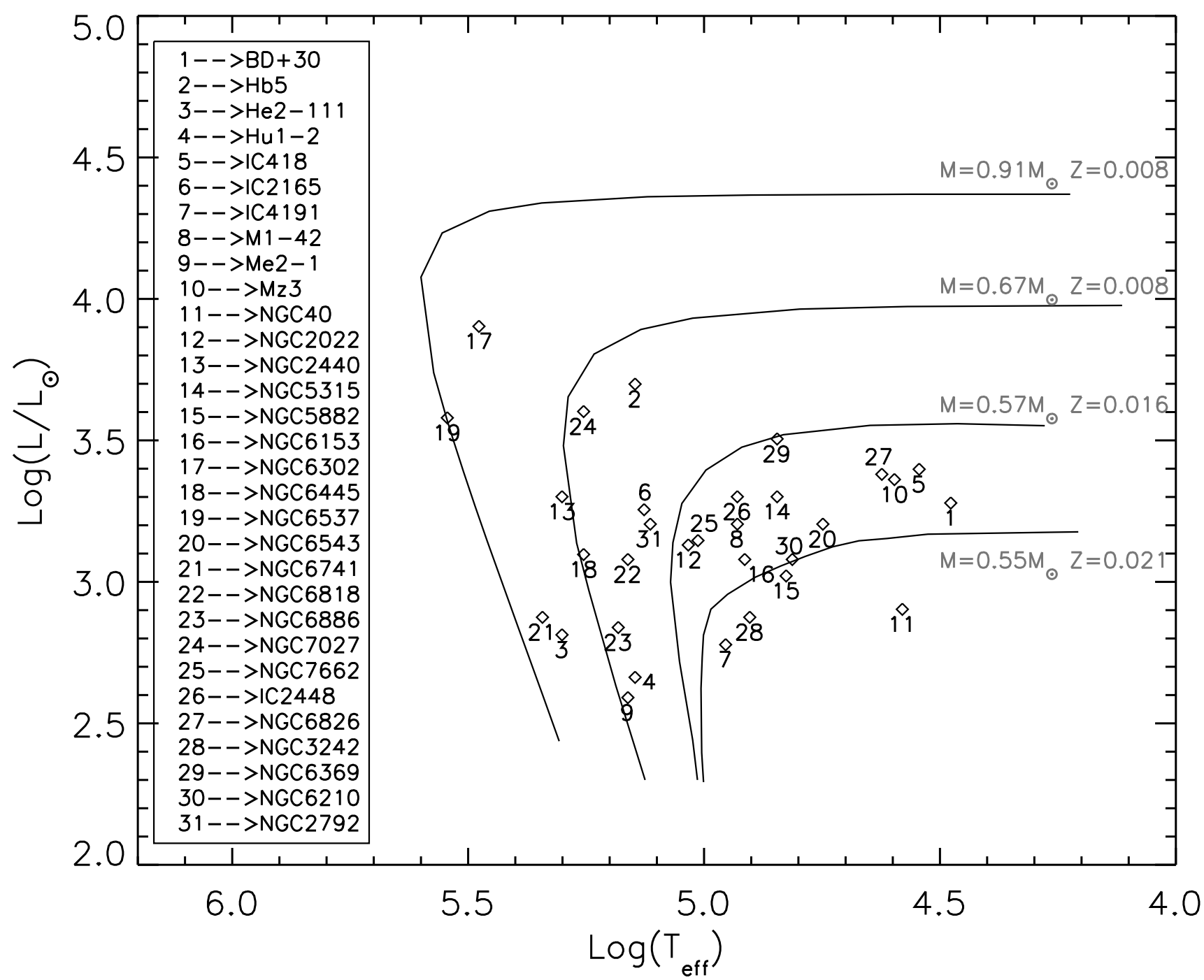

Fig. 4. The HR diagram. The models for core masses of $0.57,0.67$ and $0.91 M_{\odot}$ are taken from Vassiliadis \& Wood (1993) while the $0.55 M_{\odot}$ model is from Schönberner (1983).

\section{Summary and conclusions}

We have determined the masses of a selection of 33 wellstudied nebulae PNe by comparing the observed nebular abundances with that predicted by the evolutionary models of Karakas (2003), which is the first systematic study of the evolution of lower mass stars. A secondary purpose is to see whether the masses determined in this way are consistent with the evolutionary tracks computed by Schönberner(1983), Blöcker (1995) and Vassiliadis \& Wood(1993). The abundances used in this comparison are helium, oxygen, nitrogen and carbon. The morphology is also considered in this comparison but in a simplified form. The PNe are divided into two categories: bipolar and elliptical (including round nebulae). The result of the comparison of He vs. N/O (Sect. 2.2) indicate four PNe have core masses greater than $0.9 M_{\odot}$; three of these nebulae are bipolar (NGC 6302, NGC 6537 and He2-111). There are also four nebulae which appear to have a slightly lower initial mass, between $0.85 M_{\odot}$ and $0.9 M_{\odot}$ and have also undergone hot-bottom burning. Again three of these PNe are bipolar (NGC 2440, Hb5 and Hu1-2). Thus 6 of the 7 bipolar nebular in our selection have abundances which indicate that they originated from initially high mass stars. The only bipolar nebula which appears to be an exception is NGC 6445 which has a high $\mathrm{He} / \mathrm{H}$ ratio but a low N/O ratio which is difficult to understand on the basis of the models of Karakas.

NGC 5315 appears to be an elliptical PN with a high mass. There are three other elliptical nebulae which on the basis of a rather high $\mathrm{N} / \mathrm{O}$ ratio seem to originate from stars of rather high mass. These are NGC 6153, NGC 6886 and NGC 2392. A unlikely explanation is that these $\mathrm{PNe}$ are really bipolar seen edge on making them look round. Two of these PNe do not have a high $\mathrm{He} / \mathrm{H}$ ratio however, making this interpretation appear too simplified. All the other elliptical $\mathrm{PNe}$ have $\mathrm{N} / \mathrm{O}$ and $\mathrm{He} / \mathrm{H}$ ratios which do not substantially differ from solar and therefore have a core mass of less than approximately $0.7 M_{\odot}$, using the evolutionary models of Karakas (2003). We cannot determine the mass more precisely for these nebulae, except to say that in the models of Karakas those PNe with a high carbon abundance $(\mathrm{C} / \mathrm{O} \geq 1)$ have the higher mass.

An HR diagram for these nebulae was then constructed in order to see if the position of the PNe on this diagram can confirm the conclusions drawn from the comparison of the abundances with the predictions of Karakas. The effective temperature of the central stars is determined, usually with an error that is less than $10 \%$. The determination of the luminosity can be made in two ways. First by computing it from the temperature and radius of 
the central star, and secondly by finding it from the nebular emission in both the hydrogen and helium lines. The second method has the additional assumption that the nebula is "optically deep" to ionizing radiation of hydrogen or helium. We find that the luminosity determined from the ionized helium line is usually approximately equal to the luminosity found from the central star, indicating that the nebula absorbs all radiation which can doubly ionize helium. For about $30 \%$ of the nebulae the hydrogen ionizing radiation gives a lower luminosity indicating that in these cases the nebula is "optically thin" to this radiation. In this way a method has been found to determine which nebulae are "optically deep" to the various radiation fields. Probably the largest uncertainty in the luminosity is the distance determination so that a subsection is devoted to a discussion of the distances used. The resultant HR diagram is shown in Fig. 4. The high masses found from the abundances for the six bipolar PNe are consistent with their position on the HR diagram. The position of the seventh bipolar PN, NGC 6445, is consistent with it being a high mass object, leaving its rather low N/O ratio as a problem. The position of NGC 6886 is consistent with a rather high mass. Most of the elliptical PNe have positions consistent with low core masses. The biggest problems are: 1) NGC 7027, whose HR position indicates a high mass while its abundances give a low mass; 2) NGC 5315 whose position indicates a low mass while its abundance gives a high mass. M1-42 is also a problem but its distance is very poorly known and some of the observations are not very good. NGC 2392 has a different problem since the luminosity determined from the star is improbably high. The effective temperature that we have determined for this central star does not apply to the star measured in the visual. The most likely solution is that this central star is a binary and the secondary, which is unseen in the visual, is of a much higher temperature and is responsible for the high degree of ionization found. The possible presence of a hotter star has been suspected earlier; Ciardullo et al. (1999) have looked for it but have found only a very faint nearby star. If this is the source of ionization it cannot be a main sequence star because if this was so it would be too distant to be associated with the nebula. But the ionization source could be a star which is too close to the bright star to be observed.

In general it appears that the initial masses as determined from the observed abundances in conjunction with the models of Karakas (2003) are consistent with the initial masses predicted using the evolutionary models of Schönberner(1983), Blöcker (1995) and Vassiliadis \& Wood (1993). But being consistent is only a first step and both the models and the observed abundances should both be improved. Furthermore the various cases which appear to give inconsistent results must be understood before we can speak of agreement.

\section{References}

Asplund, M., Grevesse, N., Sauval, A. J., \& Scott, P. 2009, ARA\&A, 47, 481 Bernard-Salas, J., Pottasch, S. R., Beintema, D. A., \& Wesselius, P. R. 2001, A\&A, 367, 949

Bernard-Salas, J., Pottasch, S. R., Feibelman, W. A., \& Wesselius, P. R. 2002, A\&A, 387, 301

Bernard-Salas, J., Pottasch, S. R., Wesselius, P. R., \& Feibelman, W. A. 2003, A\&A, 406, 165
Blöcker, T. 1995, A\&A, 299, 755

Cahn, J. H., Kaler, J. B., \& Stanghellini, L. 1992, A\&AS, 94, 399 Ciardullo, R., Bond, H. E., Sipior, M. S., et al. 1999, AJ, 118, 488 Crowther, P. A., Morris, P. W., \& Smith, J. D. 2006, ApJ, 636, 1033 Feldman, U., \& Widing, K. G. 2003, Sp. Sci. Rev., 107, 665 Gathier, R., \& Pottasch, S. R. 1988, A\&A, 197, 266

Gathier, R., Pottasch, S. R., \& Pel, J. W. 1986a, A\&A, 157, 171 Gathier, R., Pottasch, S. R., \& Goss, W. M. 1986b, A\&A, 157, 191 Greig, W. E. 1971, A\&A, 10, 161

Greig, W. E. 1972, A\&A, 18, 70

Harris, H. C., Dahn, C. C., \& Canzian, B. 2007, AJ, 133, 631

Heap, S. R., \& Hintzen, P. 1990a, ApJ, 353, 200

Heap, S. R., Corcoron, M., Hintzen, P., et al. 1990b, in From Miras to PN, ed. M. O. Mennessier, \& A. Omont, 397

Hummer, D. G., \& Storey, P. J. 1987, MNRAS, 224, 801

Iben, I. Jr., \& Renzini, A. 1981, Phys. Proc. in Red Giants (Dordrecht: pub. Reidel)

Karakas, A. I. 2003, Thesis, Monash Univ. Melbourne

Karakas, A. I., \& Lattanzio, J. C. 2003, PASA, 20, 393

Kudritzki, R.-P., Mendez, R. H., Puls, J., et al. 1997, ed. Habing, \& Lamers, IAU Symp., 180, 64

Lattanzio, J. 2003, ed. S. Kwok, M. Dopita, \& R. Sutherland (ASP), IAU Symp., 209, 73

Manchado, A. 2003, IAU Symp., 209, 431

Manchado, A., Guerrero, M. A., Stanghellini, L., et al. 1996, Morph. Cat. PN, (IAC)

Marigo, P., Bernard-Salas, J., Pottasch, S. R., et al. 2003, A\&A, 409, 619

Martin, W. 1994, A\&A, 281, 526

Matsuura, M., Zijlstra, A. A., Gray, M. D., et al. 2005, MNRAS, 363, 628

Mellema, G. 2004, A\&A, 416, 623

Mendez, R. H., Kudritzki, R. P., Herrero, A., et al. 1988, A\&A, 190, 113

Mendez, R. H., Kudritzki, R. P., \& Herrero, A. 1992, A\&A, 260, 329

Napiwotzki, R. 2006, A\&A, 451, L27

Paczynski, B. 1971, Acta. Astron., 21, 417

Pauldrach, A. W. A., Hoffmann, T. L., \& Mendez, R. H. 2004, A\&A, 419, 111

Peimbert, M. 1978, IAU Symp., 76, 215

Peimbert, M., \& Torres-Peimbert, S. 1983, IAU Symp., 103, 233

Phillips, J. P. 2003, MNRAS, 344, 501

Pottasch, S. R. 1984 (Dordrecht: Reidel), Plan. Neb., 105

Preite-Martinez, A., \& Pottasch, S. R. 1983, A\&A, 126, 31

Pottasch, S. R., \& Acker, A. 1989, A\&A, 221, 123

Pottasch, S. R., \& Beintema, D. A. 1999, A\&A, 347, 974

Pottasch, S. R., \& Bernard-Salas, J. 2008, A\&A, 490, 715

Pottasch, S. R., \& Surendiranath, R. 2005a, A\&A, 432, 139

Pottasch, S. R., \& Surendiranath, R. 2005b, A\&A, 444, 861

Pottasch, S. R., Preite-Martinez, A., Olnon, F. M., et al. 1986, A\&A, 161, 363

Pottasch, S. R., Beintema, D. A., \& Feibelman, W. A. 2000, A\&A, 363, 767

Pottasch, S. R., Beintema, D. A., Bernard-Salas, J., \& Feibelman, W. A. 2001, A\&A, 387, 684

Pottasch, S. R., Beintema, D. A., Bernard-Salas, J., et al. 2002, A\&A, 393, 285

Pottasch, S. R., Beintema, D. A., Bernard-Salas, J., \& Feibelman, W. A. 2003, A\&A, 409, 599

Pottasch, S. R., Beintema, D. A., Bernard-Salas, J., \& Feibelman, W. A. 2004, A\&A, 423, 593

Pottasch, S. R., Beintema, D. A., \& Feibelman, W. A. 2005, A\&A, 436, 953

Pottasch, S. R., Bernard-Salas, J., \& Roellig, T. L. 2009, A\&A, 499, 249

Preite-Martinez, A., Acker, A., Koppen, J., et al. 1991, A\&AS, 88, 121

Renzini, A., \& Voli, M. 1983, A\&A, 94, 175

Sabbadin, F. 1986, A\&AS, 64, 579

Sabbadin, F., Benetti, S., Cappellaro, E., et al. 2005, A\&A, 436, 549

Schönberner, D. 1983, ApJ, 273, 708

Schönberner, D., Jacob, R., \& Steffen, M. 2005, A\&A, 441, 573

Stanghellini, L., Corradi, R. L. M., \& Schwarz, H. E. 1993, A\&A, 276, 463

Stanghellini, L., Shaw, R. A., \& Villaver, E. 2008, ApJ, 689, 194

Stoy, R. H. 1933, MNRAS, 93, 588

Terzian, Y. 1997, ed. H. J. Habing, \& H. Lamers, IAU Symp., 180, 29

Tylenda, R., Siodmiak, N., Gorny, S. K., et al. 2003, A\&A, 405, 627

Vassiliadis, E., \& Wood, P. R. 1993, ApJ, 413, 641

Vassiliadis, E., \& Wood, P. R. 1994, ApJS 92, 125

Wolff, M. J., Code, A. D., \& Groth, E. D. 2000, AJ, 119, 302

Zijlstra, A. A., Hoof, P. A. M., \& Perley, R. A. 2008, ApJ, 681, 1296 